\title{
LOS DISTRITOS ECONÓMICOS EN LA CIUDAD AUTÓNOMA DE BUENOS AIRES COMO NUEVA FORMA DE INTERVENCIÓN URBANA
}

\section{THE ECONOMIC DISTRICTS IN THE AUTONOMOUS CITY OF BUENOS AIRES AS A NEW FORM OF URBAN INTERVENTION}

\author{
Rodrigo Carmona ${ }^{1}$
}

\section{Resumen}

El presente trabajo tiene como objetivo central analizar la política de los Distritos Económicos en la Ciudad Autónoma de Buenos Aires, en tanto nueva forma de de intervención urbana sobre la base de la promoción de actividades con eje mayormente en la industria creativa. Los núcleos medulares de la intervención se sustentan fundamentalmente en: i) la transformación de viejos mercados o firmas industriales en áreas temáticas de promoción; ii) la convocatoria al sector privado por parte del Estado que actúa como facilitador del mercado; iii) la habilitación de una serie de incentivos económicos y acciones de fomento a las economías de aglomeración, con procesos de valorización inmobiliaria; iv) la escasa consideración de los intereses de la ciudadanía que reside en los ámbitos urbanos intervenidos. A partir de ello, se abordan los principales elementos conceptuales respecto a los debates sobre las ciudades, los procesos de transformación socio territorial y los modelos de intervención urbana en un marco de globalización y predominio neoliberal. Seguidamente, se dan cuenta de los aspectos metodológicos y se analizan las principales particularidades e implicancias políticas y sociales que asumen los Distritos como nueva forma de intervención urbana a partir de una lógica facilitadora del mercado al interior de la ciudad.

Palabras clave: Distritos Económicos, intervención urbana, implicancias políticas y sociales

\begin{abstract}
The main objective of this work is to analyze the policy of the Economic Districts in the Autonomous City of Buenos Aires, as a new form of urban intervention based on the promotion of activities with a focus on the creative industry. The central nuclei of the intervention are based mainly on: i) the transformation of old markets or industrial firms in thematic areas of promotion; ii) the call to the private sector by the State acting as market facilitator; iii) the provision of a series of economic incentives and promotion actions to agglomeration economies, with real estate valuation processes; iv) the poor consideration of the interests of the citizenship residing in the intervened urban areas. From this, the main conceptual elements regarding the debates on the cities, the processes of socio-territorial transformation and the models of urban intervention in a context of globalization and neoliberal dominance.. Next, they realize the methodological aspects and analyze the main peculiarities and political and social implications assumed by the Districts as a new form of urban intervention based on a logic that facilitates the market within the city.
\end{abstract}

Keywords: Economic Districts, urban intervention, political and social implications

\footnotetext{
${ }^{1}$ Posdoctor en Ciencias Humanas y Sociales, Facultad de Filosofía y Letras -Universidad de Buenos Aires. E-mail: rcarmona@ungs.edu.ar
} 


\section{INTRODUCCIÓN}

El presente trabajo tiene como objetivo central examinar en la Ciudad Autónoma de Buenos Aires (CABA) analizar la política de los Distritos Económicos en la Ciudad Autónoma de Buenos Aires, en tanto nueva forma de de intervención urbana sobre la base de la promoción de actividades con eje mayormente en la industria creativa. Los núcleos medulares de la intervención se sustentan fundamentalmente en: i) la transformación de viejos mercados o firmas industriales en áreas temáticas de promoción; ii) la convocatoria al sector privado por parte del Estado que actúa como facilitador del mercado; iii) la habilitación de una serie de incentivos económicos y acciones de fomento a las economías de aglomeración, con procesos de valorización inmobiliaria; iv) la escasa consideración de los intereses de la ciudadanía que reside en los ámbitos urbanos intervenidos. A partir de ello, se busca examinar los conflictos asociados a este tipo de intervención urbana como las dificultades para conciliar la acción del Estado, la dinámica del capital y la garantía de derechos sociales en las ciudades.

En este sentido, el trabajo se organiza del siguiente modo. En una primera parte, se abordan los principales elementos conceptuales respecto a los debates sobre las ciudades, los procesos de transformación socio territorial y los modelos de intervención urbana en un marco de globalización y predominio neoliberal. Seguidamente, se dan cuenta de los aspectos metodológicos y se analizan las principales particularidades e implicancias que asumen los Distritos como nueva forma de intervención urbana dentro de una lógica facilitadora del mercado al interior de la ciudad. En último término, se hacen unas consideraciones finales sobre las problemáticas consideradas.

\section{CIUDADES, TRANSFORMACIONES SOCIO TERRITORIALES Y MODELOS DE INTERVENCIÓN URBANA}

Las ciudades conforman a escala planetaria un actor preponderante del capitalismo contemporáneo por su peso demográfico, político, cultural, social y económico. Los procesos simultáneos de globalización económica, concentración de la población en grandes urbes y dinámicas de cambio socioculturales, replantean las funciones y el peso específico de estos espacios y las formas de intervención urbana. Al interior de las ciudades se desarrollan así una multiplicidad de interacciones, conflictos y procesos sociales y políticos complejos, con actores que presentan diversas lógicas, capacidades e intereses. Las ciudades componen así una construcción colectiva variada, contradictoria y en permanente transformación. La 
consideración de la ciudad en cuanto espacio de soportes materiales y funcionales que responden a diversas necesidades -sean las mismas asociadas a la sociedad o al mercado-, pone a la gestión y política urbana como objeto de disputa.

En este sentido, es posible identificar distintas concepciones de ciudad que históricamente se van modificando conforme a múltiples dimensiones: a una base estructural (socio-económica), territorial, y a las estrategias de representación y construcción de lugar que los actores sociales sean locales como no locales (PÍREZ, 1995 y 2008) (CARMONA, 2015). Diversos autores coinciden en señalar que la reestructuración del sistema capitalista actual exhibe como rasgos dominantes la preeminencia de la tecnociencia, de la información y conocimiento y de las finanzas (DE MATTOS, 2010) (SILVEIRA, 2008) (SANTOS, 2000). El concepto de globalización destaca una división social y territorial del trabajo nunca antes desplegada, en tanto que las empresas atraviesan las fronteras nacionales $y$, sobre la base de los factores de localización, eligen distintos lugares del planeta para desarrollar las actividades relacionadas a su producción.

Estas transformaciones hacen patente estructuralmente un cambio en la participación, involucramiento y responsabilidad estatal (BRENNER \& THEODORE, 2002) (HARVEY, 2007) mediante la destrucción, desarticulación y deslegitimación de políticas e instituciones propias del Estado de Bienestar. Ello determina la conformación y consolidación de nuevas intervenciones estatales para facilitar la participación privada con fines de negocios, como así también la privatización y desregulación de activos públicos. El Estado dejar de actuar sobre el territorio para pasar a cumplir un rol de tipo subsidiario en tanto acondicionador y promotor de las transformaciones del espacio urbano, controlado mayormente por estrategias empresariales. De este modo, se establecería un cambio en el modo de gestión urbana con eje en una merma de la intervención y la inversión estatal, ubicando al capital privado en el centro del desarrollo y la configuración urbana en una dinámica neoliberal de producción de la ciudad (DE MATTOS, 2002) (CICOLLELLA, 2011) (ZAPATA, 2017).

En este marco, tal como resalta (CARRIÓN, 2007), es posible apreciar distintos modelos de intervención urbana. Un modelo remite a un enfoque mercantil privado y concibe al manejo público-estatal como un limitante para el desarrollo urbano, mientras que el otro le asigna un mayor peso a lo ciudadano con eje en un enfoque de derechos. Estos dos modelos no se muestran de manera pura, aunque puede apreciarse el predominio de uno sobre el otro. El primero es el de la «ciudad empresarial-privada» como mecanismo que difunde y generaliza los principios de mercado en un contexto de demanda disgregada y atomización de los conflictos 
urbanos. Se critica la intervención estatal por ineficiente y centralizadora de las decisiones, frente a los beneficios delo privado en términos de eficiencia y mayor participación de la ciudadanía pensada en forma privatista y desde una lógica de "cliente". En el plano administrativo, con un alcalde pensado como gerente se propone una reestructuración del aparato municipal mediante tercerizaciones de los servicios y beneficios a sectores específicos. Esta dinámica privilegia la gestión privada sobre la pública, y modifica además la relación entre el Ejecutivo y el concejo municipal, lo cual perjudica la representación y una visión integral del conjunto al concentrar las decisiones en una fracción alejada de la sociedad. De esta forma, se busca «despolitizar» las decisiones relevantes de política urbana otorgando mayor injerencia a grupos privados de modo de lograr un manejo presupuestario más eficiente y dotar de mayor gobernabilidad a la gestión municipal.

El otro modelo alternativo, que el autor denomina «ciudad inclusiva», busca resignificar lo público como una opción frente a los problemas urbanos mediante distintas estrategias. En primer término, sobre la base del restablecimiento del aparato gubernamental pensado como instancia amplia, dentro del cual la participación y la representación son elementos centrales. Por otra parte, a partir de la reconstitución de la ciudad como espacio público pensado como factor estructurante de la ciudad tanto en su dimensión física (organización espacial), social (identidades e integración) y ciudadana (constructor de derechos: salud, educación, etc.). Se destacan así las ideas de democratización del gobierno y de racionalización de su administración pública poniéndose el énfasis en lo territorial por sobre lo sectorial. Este enfoque busca afianzar la gobernabilidad y el desarrollo urbano (económico, social, cultural), mediante una mejor integración social y politización de su funcionamiento.

La reestructuración económica mundial iniciada en los años '70 y consolidada en la década de los '90 con una mayor flexibilización productiva, la menor gravitación del movimiento obrero junto a la apertura económica de la mayoría de los países y el aumento de los flujos de capitales globales, determinó un posicionamiento mayoritario de la gestión urbana sobre la base de una concepción de ciudad con eje en el interés privado. La importancia puesta en la atracción de inversiones y mejoras en las condiciones del territorio como pilar del denominado empresarialismo urbano, aparece señalado hace varios años por (HARVEY, 1989). (THEODORE, PECK \& BRENNER, 2009), precisamente, plantean estos cambios a partir de la decreciente rentabilidad de las industrias de producción masiva y de la crisis propia del Estado de Bienestar. (DE MATTOS, 2010), por su parte, destaca también el papel jugado en este sistema por parte de las nuevas tecnologías de la información y las comunicaciones además de la extensión de la 
liberalización económica. Esta reestructuración impactó, en términos territoriales, en la consolidación global de nuevos espacios transnacionales de actividad económica que modificaron las condiciones en las que tuvo lugar la producción, el intercambio y el consumo de bienes y servicios

La extensión de valores como el individualismo y la competitividad fueron elementos relevantes del sistema ideológico hegemónico, que iría permeando de forma generalizada la vida cotidiana de las personas en todos los planos. En cada país y contexto particular, las diversas relaciones de fuerza entre los grupos sociales establecieron distintos impactos en relación a las políticas instrumentadas, en el marco de un proceso que tuvo alcance global. El planeamiento urbano, en tanto técnica del mundo de los negocios, se convirtió así en una de las principales herramientas para enfrentar los problemas de las ciudades. Ello convivió con aumentos de los niveles de segregación urbana, en un dinámica dual mayormente caracterizada por la elitización de los barrios centrales y la metropolización de la clase trabajadora (MOLLENKOPF\& CASTELLS, 1991) (DE MATTOS, 2002) (MONTESINOS, 2016).

El modelo de apertura económica y las transformaciones planteadas sentaron las bases de un nuevo ordenamiento urbano. Se establece así una relación directa entre la destrucción parcial de la institucionalidad vigente a través de iniciativas reformadoras orientadas al mercado y la creación de infraestructuras para facilitar el crecimiento económico orientado al mercado, la mercantilización de bienes y servicios y una normatividad con eje en el capital. Ello determina como dinámica asociada el despliegue de procesos de renovación urbana derivados del deterioro de las infraestructuras urbanas y, también en ciertos casos, la pérdida de población en el territorio (SMITH, 1996) (CARRIÓN, 2005) (RODRÍGUEZ \& DI VIRGILIO, 2014).

En este escenario, se inserta el modelo de "ciudad creativa" sugerido por (FLORIDA, 2002 y 2005) que incentiva la clusterización o concentración geográfica de las industrias creativas, bajo el postulado que la cercanía física y la aglomeración de agentes, firmas e instituciones en ámbitos con ciertas particularidades se presenta positivo a nivel urbano y productivo. Tras la fuerte acogida que generaron estos escritos, en los siguientes años la gestión de la creatividad fue uno de las temáticas más enfatizadas en el campo de la planificación urbana.

Como bien destaca (MONTESINOS, 2016:9): "La ciudad creativa fue uno de los proyectos que se concibió dentro de la lógica neoliberal para dar respuesta a la pregunta de cómo debía estructurarse la ciudad del futuro. De hecho, se trata de uno de los paradigmas que se elaboraron para dotar de significado a un concepto que carecía de él, porque se definía por 
oposición al periodo inmediatamente anterior: la ciudad postindustrial. Aunque cada uno de ellos -la ciudad informacional, la ciudad del conocimiento, la ciudad inteligente...- tiene sus propios matices, comparten una característica básica, que es la adaptación espacial a la reestructuración de la economía urbana, y el establecimiento de una nueva economía basada cada vez más en lo que vagamente se ha definido como la dimensión inmaterial de la producción y el consumo. Con el paso del tiempo se ha visto que ninguno de estos paradigmas ha dominado por encima de los demás, sino que la mayoría de las veces son complementarios y el énfasis con el que se aplican en cada ciudad depende en función de las necesidades de sus políticas urbanas".

De esta forma, el despliegue de industrias culturales y creativas fue posicionándose a escala mundial como tema relevante en la agenda política de las ciudades. El diseño de nuevos polos culturales y el desarrollo de grandes eventos como productos de un nuevo marketing urbano, permitió generar importantes niveles de aceptación ciudadana en un contexto de promoción de la ciudad creativa. Ello, no obstante, lejos de cuestionar el paradigma vigente se ve enmarcado en una dinámica más amplia neoliberal que respecto al período anterior, provoca mayores niveles de fragmentación social y desigualdad al interior de las distintas ciudades.

\section{ABORDAJE METODOLÓGICO Y PRINCIPALES CONSIDERANDOS}

En este trabajo se busca analizar la intervención urbana en tanto política pública mediante la consideración de tres dimensiones: como discurso, contemplando su contenido significante; como curso de acción, esto es, el conjunto de decisiones y técnicas que se desarrollan y son pertinentes y relevantes en un marco específico de implementación; y las políticas consideradas en tanto entramado de actores, lo que lleva a identificar los actores y sus tomas de posición en cada momento del proceso (DI VIRGILIO, 2010). En estos términos, concebimos a la política pública como un conjunto de acciones y omisiones que expresan la intervención de distintos actores, entre ellos el Estado, que se movilizan en relación a una cuestión, entendida la misma como un asunto socialmente problematizado (OSZLAK \& O’DONNELL, 1981). El modo en el que el Estado, junto a los diversos actores, se implica en el procesamiento de las cuestiones plantea distintas tomas de posición (que no son siempre homogéneas, ni constantes en el tiempo) en el marco del proceso de la política pública.

Para el despliegue de esta tarea, en primer término, se hace un análisis de distintas fuentes documentales (normativa, artículos periodísticos y académicos, discursos de 
funcionarios públicos de las áreas pertinentes, imágenes, videos, etc.) de modo de dar cuenta de las particularidades que asumen los Distritos como nueva forma de intervención urbana. Ello se complementa, en segundo lugar, con la realización de algunas entrevistas en profundidad con distintos informantes clave involucrados en esta política. Al respecto, se consideran particularmente dos casos distintivos: el "Distrito Audiovisual" en la zona norte de Buenos Aires, y el "Distrito de la Artes" en la zona sur de la ciudad.

\section{LOS "DISTRITOS" COMO POLÍTICA EN LA CIUDAD AUTÓNOMA DE BUENOS AIRES}

Los "Distritos", denominados inicialmente creativos y en la actualidad económicos, aparecen desde el discurso del gobierno porteño $\mathrm{PRO}^{2}$ como un nuevo tipo de intervención urbana, a favor de pensar la ciudad como espacios temáticos asociados a una actividad industrial en pos de favorecer según los funcionarios el desarrollo barrial (fundamentalmente de la zona sur, el área más postergada de la CABA).

Los distritos contemplan así la delimitación de un espacio territorial determinado, en el cual se establecen incentivos para la promoción de una industria estratégica específica, concentrando en un lugar a empresas del mismo sector, y al mismo incentivando el desarrollo de un barrio previamente olvidado. Dentro de los incentivos se pueden considerar créditos y exenciones impositivas, como la creación de una importante base de infraestructura que atraiga a las empresas del sector.

La política de distritos parte de la pretensión de las autoridades locales de recuperar espacios no aprovechados y se referencia en experiencias internacionales de clusters (como Silicon Valley en California y el distrito Barcelona 22@), más allá de las claras diferencias y especificidades propias de estas experiencias. La concentración espacial de actividades "creativas", el impulso público para acondicionar el entorno y apalancar al sector privado, junto a los incentivos fiscales y regulatorios en pos de impulsar el desarrollo local, constituyen elementos centrales de su despliegue. Se plantea entonces la importancia de producir mercado -del suelo, comercial, industrial- donde no lo hay, con un fuerte impulso del actor estatal. ${ }^{3}$

\footnotetext{
2 En los años 2007, 2011 y 2015 el PRO (propuesta Republicana) ganó la Jefatura de Gobierno de la Ciudad Autónoma de Buenos Aires, con las candidaturas del empresario y actual presidente Mauricio Macri (con dos mandatos, 2007-2011 y 2011- 2015) y su sucesor, Horacio Rodríguez Larreta (con mandato, 2015-2019). El PRO se caracterizaría por una orientación política de cuño neoliberal y promercado respecto a las principales problemáticas de la ciudad.

${ }^{3}$ Ver al respecto (SOCOLOFF, 2017).
} 
Se parte así de una visión que le da importancia al agrupamiento de instituciones y personas en un ámbito local, buscando generar sinergia entre las empresas y la comunidad, asociada a un modo de vida. La política de distritos, desde los funcionarios del Gobierno de la CABA busca partir del diagnóstico de las diferencias internas de la Ciudad, para promover zonas de menor desarrollo. Además, se buscaban aprovechar las potencialidades de una ciudad de servicios a través de la promoción de industrias estratégicas.

\section{Figura 1. Distritos de la Ciudad Autónoma de Buenos Aires}

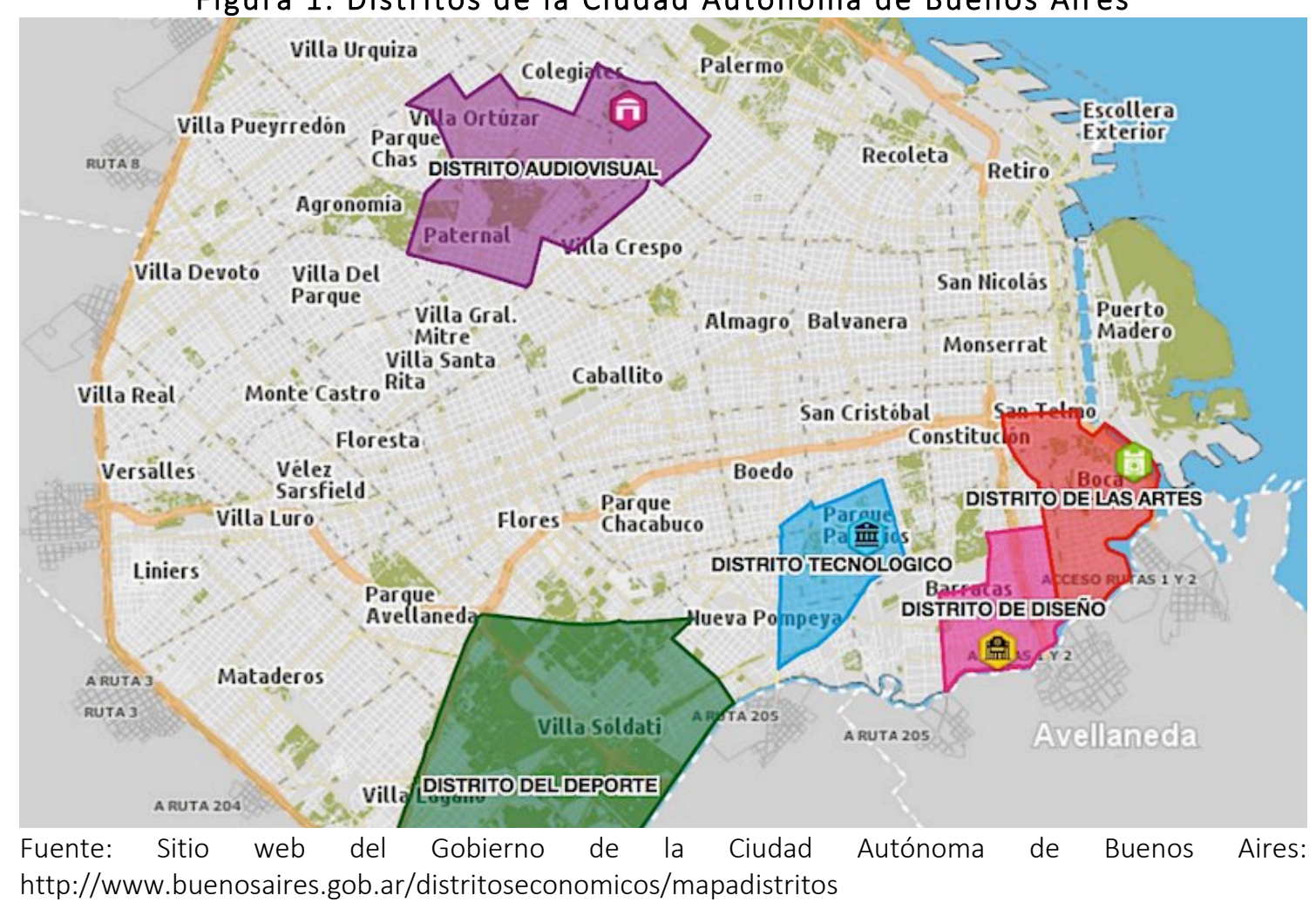

A partir de su definición, conforma una política económica con fuertes efectos urbanos, puesto que busca promover la reconversión de usos del suelo en áreas bien ubicadas de la ciudad. En efecto, según sostiene el Ministro de Desarrollo Económico de la ciudad Francisco Cabrera: "Nuestro objetivo es promocionar las industrias estratégicas de la Ciudad, entre ellas, audiovisual, moda y diseño que es lo que hace que el mundo mire a Buenos Aires. Este tipo de iniciativas junto con el desarrollo de los distritos (tecnológico, audiovisual, diseño y artes) generan trabajo y convierten a Buenos Aires en una ciudad más competitiva". ${ }^{4}$

El primero de los Distritos que se crea, es el Tecnológico, en el año 2008 mediante la Ley № 2972 y se sitúa en 200 hectáreas de los barrios de Boedo, Parque de los Patricios y

\footnotetext{
${ }^{4}$ Gacetilla del Ministerio de Desarrollo Económico de la Ciudad Autónoma de Buenos Aires, 22/08/2012.
} 
Pompeya. Su desarrollo parte de un conjunto de desgravaciones impositivas a empresarios e inversores del área de las telecomunicaciones y las nuevas tecnologías de la información producción de software o hardware- interesados en radicarse en el territorio delimitado. Las empresas que se instalan, reciben los siguientes beneficios: menor costo de alquiler, menor carga impositiva, concentración de recursos humanos reales (profesionales) y potenciales (estudiantes), incentivos fiscales y de infraestructura, exención del impuesto a ingresos brutos y $A B L$-alumbrado, barrido y limpieza (por 15 años para empresas nacionales y 10 años para internacionales), exención del impuesto de sellos, exención del pago del derecho de delineación y construcciones (por 10 años), líneas de crédito preferenciales del Banco Ciudad y subsidios para la obtención de certificaciones de calidad. El Distrito Tecnológico cuenta con más de 200 empresas radicadas y durante este tiempo, generó más de 11 mil empleos vinculados a la tecnología. ${ }^{5}$

Desde su creación, el gobierno desplegó diversas inversiones y obra pública para revitalizar la zona: i) mejoramiento del Parque de los Patricios: más de 10 mil metros cuadrados de veredas, regularización de 440 puestos en la feria de artesanos, 3 patios de juegos nuevos, 1 cancha multipropósito para realizar deportes, más de 100 luminarias nuevas, 190 bancos de plaza, más de 150 nuevos cestos de basura y plantación y poda de 8 mil arbustos; ii) inversión en la vía pública: arbolado (832 unidades), alumbrado (496 luminarias), veredas (14.400 m2) y bacheo y demarcación (3000 m2); seguridad: instalación de una Comisaría de la Policía Metropolitana ( $\left.\mathrm{N}^{\circ} 4\right)$ con cobertura en la Comuna 4, dotada de 24 móviles y 430 efectivos; iii) transporte: consolidación de la línea $\mathrm{H}$ de subte y el Metrobus Sur; cuenta con 3 estaciones de la línea $\mathrm{H}$ (Caseros, Parque de los Patricios y Hospitales) ${ }^{6}$.

Entre las acciones pendientes del distrito figuran la creación del Centro Metropolitano de Tecnología (CMT), que funcionará en dos manzanas dentro del Distrito. Tendrá instalaciones para empresas de comunicaciones y tecnología, oficinas para el Gobierno de la Ciudad Autónoma de Buenos Aires, instituciones universitarias privadas (ITBA, USAL, CAECE), áreas comunes, un auditorio para 300 personas, aulas disponibles para las empresas y salas de reuniones. "Traer las universidades al Distrito Tecnológico ya es una realidad. A la revitalización

\footnotetext{
${ }^{5}$ Ver para su análisis (DÍAZ, FERME \& RASPALL, 2011), (SOCOLOFF ET AL 2012) (GOICOCHEA 2012 y 2014).

${ }^{6}$ Información del Gobierno de la Ciudad Autónoma de Buenos Aires.
} 
que vive Parque Patricios ahora se le suma la oferta académica", según lo declarado desde el Ministerio de Desarrollo Económico porteño?

Posteriormente, en el año 2011 mediante la aprobación de la Ley № 3876 se constituye el Distrito Audiovisual. El mismo ocupa una superficie de 720 hectáreas e incorpora los barrios de Chacarita, Villa Ortúzar, Paternal y partes de Colegiales y Palermo. Tiene como objeto impulsar la industria audiovisual permitiendo el establecimiento de empresas y productoras de cine, televisión, publicidad y animación, a las que se les dan -al igual que el Distrito Tecnológicoexenciones impositivas. Desde su creación, incluye alrededor de 175 empresas concentradas a nivel geográfico interconectadas junto a, suministradores especializados, proveedores e instituciones vinculadas al sector. El Mercado del Dorrego, situado en el corazón del Distrito Audiovisual, es el lugar que concentra todas las oficinas del gobierno de la ciudad para el estímulo de esta industria.

Hacia fines del año 2012, mediante la Ley № 4353, se conforma el Distrito de las Artes con 300 hectáreas aproximadamente. Situado fundamentalmente en el barrio de La Boca y también en parte de Barracas y San Telmo, busca posicionar al sector artístico como motor económico de una zona con fuerte identidad cultural. La creación del Distrito ofrece incentivos para favorecer el desarrollo de mayor infraestructura para las artes, la promoción de nuevas actividades culturales y nuevos puestos de trabajo. Es una zona libre de impuestos para el sector, y además tiene líneas de créditos preferenciales para los nuevos establecimientos culturales que se muden allí.

Este Distrito ya cuenta con varios lugares de interés cultural como la Fundación Proa y dos nuevos faros de la actividad cultural como la Usina del Arte y el Museo de Arte Moderno. Incluye todas las artes visuales: pintura, escultura, grabado, fotografía, arte digital; artes musicales, tanto popular como académica; artes literarias y las artes escénicas (teatro, danza, ópera, murgas, títeres, etc.).

El Distrito del Diseño, por su parte, ocupa 230 hectáreas en el barrio de Barracas. Aunque la normativa (ley 4761) se aprueba hacia fines del año 2013, el Gobierno de la Ciudad Autónoma de Buenos Aires presenta al área especializada en la temática desde la inauguración del Centro Metropolitano del Diseño en 2005 (el cual funciona en un antiguo edificio donde funcionaba el Mercado del Pescado de Barracas). Desde ese lugar se realiza en forma anual el Festival Internacional del Diseño y se promueve la radicación de empresas y profesionales del

\footnotetext{
7 Diario PERFIL:“En Parque Patricios apuestan a la tecnología y en La Boca, al arte”, 11/09/2015.
} 
campo del diseño gráfico, textil, de indumentaria e industrial, con similares beneficios fiscales que en los distritos previos. Los objetivos fundamentales de la legislación asociada son: i) plantear un horizonte temporal determinado de 15 años, para incentivar al sector en el largo plazo; ii) dar respuestas a las necesidades de la industria, actuando como el mayor polo generador de creación y producción de diseño; iii) promocionar la residencia de empresas y profesionales del sector en Barracas, otorgando beneficios fiscales tales como exenciones impositivas y tributarias.; iv) facilitar créditos del Banco Ciudad, programas de incorporación de diseño y de internacionalización y acercar las diversas herramientas que ofrece el Centro Metropolitano de Diseño (CMD) para el desarrollo de todo el sector.

Finalmente, el Distrito del Deporte integra gran parte del barrio Villa Lugano y los barrios de Villa Riachuelo y Villa Soldati, con una superficie total de 21,9 km2. Fue creado a fines del año 2014 a través de la ley 5235 y se encuentra en plena construcción. Su fin es la revalorización de la Comuna 8 , a través de la promoción de las actividades de la producción e industria deportiva mediante beneficios fiscales para aquellas empresas del sector que allí se radiquen. En este caso la inversión en infraestructura es muy importante y uno de los requisitos fundamentales para que pueda crecer y generar beneficios para los barrios. Busca con su desarrollo mejoras urbanas, económicas y de opciones laborales para los vecinos

La propuesta se orienta a brindar la posibilidad de instalarse a las fábricas de artículos deportivos, de embarcaciones o las empresas de servicios orientados al deporte así como también constructores y desarrolladores. Además de las empresas que se instalarían en la zona, también se plantean diversas mejoras en el espacio público: i) Metrobus Sur: para un mejorar conexión del transporte con el Centro de Trasbordo Constitución, buscando beneficiar a 250.000 personas; ii) mejoras generales en el espacio público; iii) Saneamiento del Lago Soldati; iv) renovación y mejoras del Parque Roca; v) Ciudad del Rock: nuevo estadio Ciudad del Rock, en el predio del Parque de la Ciudad; vi) Terminal de Omnibus Dellepiane; vii) Centro de Cargas; y viii) sede de los Juegos Olímpicos de la Juventud en 2018 y construcción de la Villa Olímpica.

De esta forma, cuatro de los cinco distritos con que cuenta la Ciudad se encuentran ubicados en la zona sur: el Distrito de Diseño en Barracas, el Tecnológico en Parque Patricios, el de las Artes en La Boca, Barracas y San Telmo, y el del Deporte en Villa Lugano, Villa Riachuelo y Villa Soldati. Desde el discurso oficial, los distritos apuntan a ser una política pública de desarrollo económico con eje central en esta zona más postergada, siendo los mismos espacios temáticos que deben crecen alrededor de una industria en particular. Este despliegue, sin embargo, no se acompañado de políticas sociales y económicas más amplias e igualadoras que 
busquen integrar al sur más relegado con el resto de la ciudad. Del mismo modo, la política de los "Distritos" se plantea mayormente como una intervención urbana pro-mercado con un fuerte eje en la inversión inmobiliaria y el papel del Estado como facilitador de la inversión privada.

No obstante, tal como señalan (ARQUEROS \& GONZÁLEZ REDONDO, 2017), en cada distrito se privilegian distintas estrategias vinculadas a las características de la actividad promocionada y según el tipo de desarrollo territorial que se busca impulsar en cada zona. Por ello, mientras que el Distrito Tecnológico en Parque Patricios, privilegia la instalación de industrias limpias y construcciones sustentables, el Distrito del Diseño en Barracas impulsa empresas con capacidad de exportación y se busca el emplazamiento de emprendimientos más pequeños. El Distrito de las Artes en el barrio de La Boca, por su parte, al promoverse la actividad artística se permite que las empresas adheridas a la ley de Mecenazgo (ley N²264) puedan destinar un $15 \%$ de los impuestos al financiamiento de esa actividad o el Distrito del Deporte que instrumenta un perfil industrial más tradicional. Respecto al desarrollo inmobiliario, en los barrios de La Boca y Barracas este elemento aparece más fuertemente que en otras zonas producto de los procesos de renovación urbana que han venido atravesando en los últimos años. En relación a la promoción del empleo, los estímulos más importantes se dan por su parte en el Distrito Tecnológico y en el Distrito del Diseño.

\section{EL DISTRITO AUDIOVISUAL Y EL DISTRITO DE LAS ARTES: PRINCIPALES ACCIONES Y ACTORES IMPLICADOS}

\section{El Distrito Audiovisual}

La industria audiovisual porteña aparece en los últimos años con un crecimiento importante producto de la sanción de la Ley de Promoción de la industria audiovisual y la creación del Distrito. La sanción de esta ley se da en el marco de una tendencia general de las políticas públicas sobre industrias culturales de la Ciudad que ponen el foco principalmente sobre sus aspectos económicos.

El proyecto de ley presentado a la Legislatura por el Jefe de Gobierno fue controversial desde sus inicios, a tal punto que diputados y representantes de los principales sindicatos de la industria se congregaron para presentar proyectos alternativos. La versión última del proyecto, sin embargo, no se diferenció en líneas generales de la propuesta inicial. Aunque se convocó a representantes de sindicatos y asociaciones a participar de la elaboración y discusión, sus 
propuestas de modificación al texto original no se consideraron. Las posturas más críticas planteaban un conjunto de elementos, tales como reivindicar el carácter artístico-cultural de la producción audiovisual y la clusterización que involucra al proyecto, destacando que dentro de los límites del Distrito Audiovisual se encuentran instaladas las empresas más grandes, siendo las mismas beneficiadas de incentivos que en principio no precisan. Otro aspecto objetado, se vincula con el negocio inmobiliario asociado que podría tender a una reconversión urbana y el posible desplazamiento de la población de menores recursos. Por último, la incorporación de empresas de distribución y exhibición entre las beneficiarias también suscitó críticas puesto que las que dominan estos rubros son principalmente transnacionales ${ }^{8}$.

Al respecto, un inversor inmobiliario daba cuenta del proceso desplegado: "El Distrito Audiovisual de Colegiales crece cada día más; muchas productoras se instalaron en la zona no sólo por sus beneficios impositivos, sino también por su privilegiada ubicación dentro de capital”. De esta forma, las ventajas de localización además de los incentivos otorgados para la instalación de grandes jugadores de la industria audiovisual constituían elementos claves de la dinámica desarrollada.

En el sitio de la Ciudad Autónoma de Buenos Aires, el mapa del Distrito Audiovisual daba cuenta de las empresas por fuera del Distrito como dentro de sus límites (figura 1).

Figura 2: Distrito Audiovisual

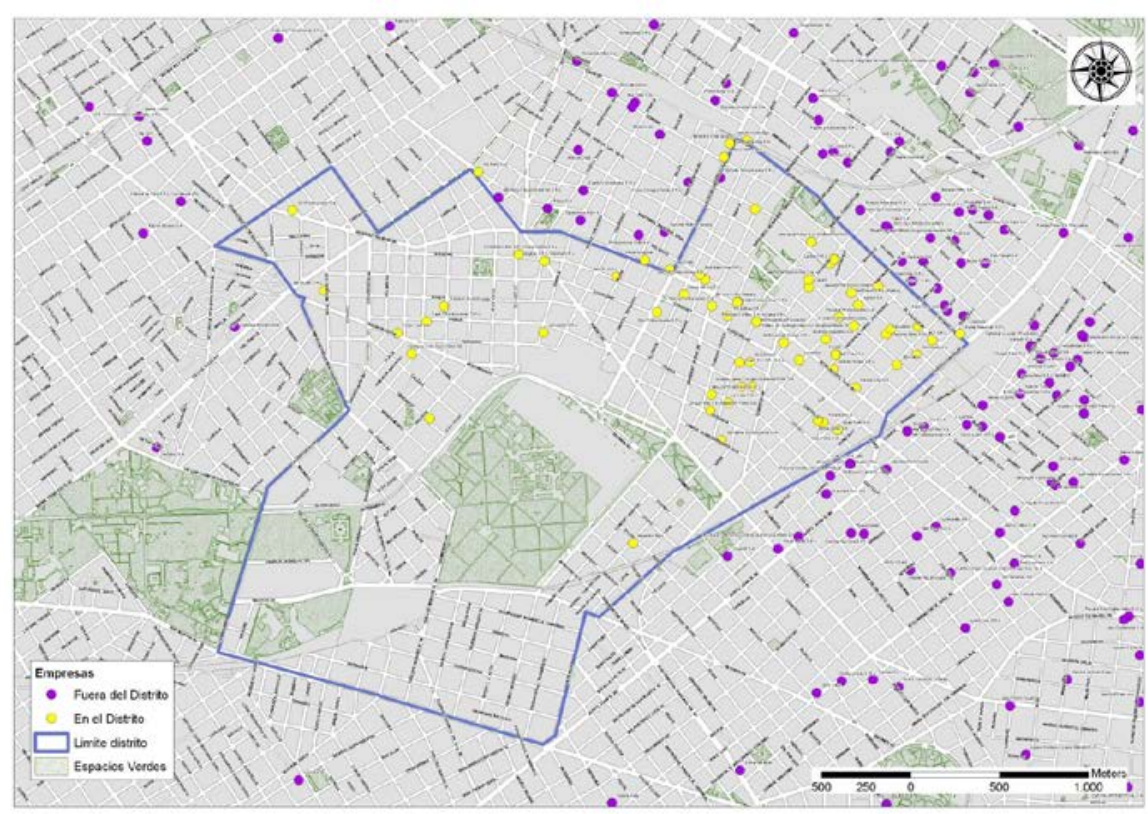

Fuente: Sitio web del Gobierno de la Ciudad Autónoma de Buenos Aires http://audiovisual.mdebuenosaires.gov.ar/contenido/editor/Image/Mapa\%20Distrito\%20Audiovisual.jpg

${ }^{8} \operatorname{Ver}($ GIONCO, 2012) y (GONZÁLEZ, 2014). 
En estos términos, el crecimiento del Distrito hasta el año 2015 podía apreciarse a partir de las siguientes cifras:

1. Las empresas nuevas radicadas en el distrito audiovisual desde la reglamentación de la ley eran: 7

2. Las empresas radicadas que ya estaban funcionando dentro de los límites del distrito y que se inscribieron en el registro para percibir los beneficios de la ley eran: 43

3. Las empresas radicadas en CABA por fuera de los límites del Distrito Audiovisual y registradas para percibir los beneficios de la Ley eran: 26

4. Empresas inscriptas en el REA del distrito audiovisual eran: 3

Sobre esta base, un análisis de las producciones audiovisuales que solicitaron permisos para filmar en espacios públicos de la Ciudad de Buenos Aires $^{9}$ mostraba en líneas generales un mantenimiento en la cantidad de emprendimientos realizados (cuadro 1).

\section{Cuadro 1. Producciones audiovisuales que solicitaron permisos para filmar en espacios públicos de la Ciudad de Buenos Aires (años 2014 y 2015)}

\begin{tabular}{|c|c|c|}
\hline & 2014 & 2015 \\
\hline Publicidad & 314 & 326 \\
\hline Largometrajes & 45 & 49 \\
\hline Estudiantes & 74 & 78 \\
\hline Programas de TV & 51 & 55 \\
\hline Cortometrajes & 10 & 8 \\
\hline Videos Clips & 7 & 5 \\
\hline Institucionales & 9 & 9 \\
\hline Documentales & 14 & 2 \\
\hline Fotografia & 32 & 32 \\
\hline Otros & 1 & 3 \\
\hline
\end{tabular}

Fuente: BASEt

La dinámica del sector y del Distrito en la Ciudad, a partir de una encuesta a productoras realizada por (BORELLO \& GONZÁLEZ, 2012), mostraba un modelo de producción flexible. La mayor parte de las empresas contrataban a terceros, buena proporción del equipamiento y de los servicios que requieren. La posesión y el mantenimiento de equipos en general costosos parecían ser no factibles. Esto se vinculaba con la naturaleza de los proyectos, el alto nivel de incertidumbre y la baja especialización en la producción, que los lleva a producir publicidades, videoclips, documentales, películas de ficción, programas de TV e institucionales,

\footnotetext{
9 Buenos Aires cuenta con una única mesa de entradas para la gestión de los permisos de rodaje en espacios públicos: BASet. Funciona con un sistema de gestión online, y además, en la Sede del Distrito Audiovisual funciona una mesa de atención para iniciar permisos de filmación.
} 
entre otras producciones. El equipamiento y los servicios eran incorporados, de este modo, no sólo según el proyecto sino también en relación al tipo de producto. En consecuencia, la diversificación de la producción hacía que los productores armasen esquemas de producción muy variables entre sí, lo cual requería una estructura flexible de organización y funcionamiento.

Por otra parte, la cuestión del vínculo con la ciudadanía en esta zona (con predominio de sectores medios) intentó ser más cuidada que en otros Distritos. La Ciudad buscó poner a disposición una comunicación directa con los vecinos en los lugares de rodajes, de modo de informar que lo que "generalmente" molesta al vecindario es una acción creativa y generadora de empleo. Con el tiempo, no obstante, las actividades del Distrito se irían mayormente aceptando por parte de los vecinos junto al desarrollo de distintas actividades recreativas proyección de películas, charlas, etc.-. El aumento de la especulación inmobiliaria en todos los barrios afectados, con nuevos edificios de departamentos, comercios y ofertas gastronómicas, iría de la mano mayormente con el alejamiento de la población de menores recursos en términos de "gentrificación" (consolidando la tendencia excluyente de décadas previas sobre la zona) y el desarrollo incipiente de la industria audiovisual en el último tiempo.

\section{Distrito de las Artes}

El Distrito de las Artes se conforma con el objeto de promover la inversión en artes visuales, literarias, musicales y escénicas de la Ciudad. Sobre la base de la oferta cultural mayormente centrada en el barrio de La Boca y relanzada con la "Usina de las Artes" (vieja usina eléctrica convertida en un importante espacio cultural y de diversas intervenciones artísticas) desde las autoridades, se postulan los beneficios que este proyecto implica: i) generación de nuevos puestos de trabajo; ii) promoción de la exportación de obras artísticas; iii) conformación de un ecosistema artístico en donde convivan los artistas, los productores, los distribuidores y comerciantes artísticos; iv) conversión del distrito en una zona de atracción turística/cultural junto al desarrollo de infraestructura asociada en la zona sur.

La Ley 4.353 que constituye el Distrito de las Artes regula entonces los beneficios fiscales que alcanzan a todos aquellos que realicen actividades culturales en el Distrito de las Artes, sean formadores, creadores, productores, gestores o difusores de todas las actividades promovidas. Incluye a personas físicas o jurídicas radicadas o quieran radicarse en el Distrito, considerando diversos tipos de beneficiarios: los que realicen en forma principal alguna de las 
actividades promovidas; desarrolladores de infraestructura artística; estudios de artistas; centros culturales y centros educativos. ${ }^{10}$

Se promueve así por ley, como sostiene THOMASZ (2016), a los artistas independientes que desenvuelvan actividades creativas (sea en espacios, talleres o respecto a los inmuebles en los que residen) pero también a otras personas que, sin ser artistas se orienten a gestionar, difundir y comercializar productos artísticos realizados por otros. Se da un tratamiento similar al artista como a otros agentes que mercantilizan, comercializan o difunden "obras artísticas", planteándose una importante ambigüedad respecto a la definición de las mismas.

Si bien el Distrito de las Artes incluye algunos sectores de San Telmo y Barracas, su mayor territorio está conformado por el barrio de La Boca (figura 2):

Figura 3. Distrito de la Artes

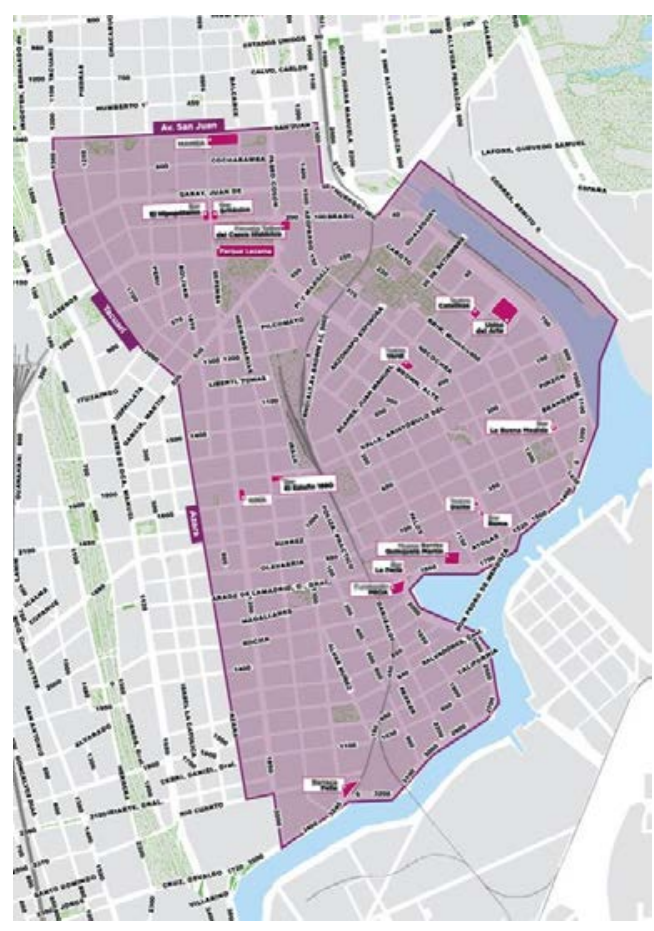

Fuente: Sitio web del Gobierno de la Ciudad Autónoma de Buenos Aires. https://twitter.com/distartes/status/271719941475028992

\footnotetext{
10 Los beneficiarios gozan de un área libre de impuestos locales por 10 años. Allí cuentan con exención de Ingresos Brutos, ABL, Sellos y Derechos de Delineación y Construcciones. Además, quienes desarrollan infraestructura artística en la zona, obtienen un crédito fiscal de Ingresos Brutos equivalente al $25 \%$ de lo invertido. Y quienes además recuperan inmuebles de valor patrimonial, amplían su beneficio a un 35\% de lo invertido. Los beneficiarios pueden acceder también a Líneas de crédito del Banco Ciudad para el financiamiento de compra, construcción y restauración de inmuebles, mudanzas y adquisición de equipamiento. También están incluidos aquellos que financian proyectos culturales en el Distrito de las Artes mediante la Ley de Mecenazgo, quienes pueden ampliar su porcentaje de deducción de Ingresos Brutos de un $2 \%$ a un $5 \%$ o 10\%, según el tipo de contribuyente que sea.
} 
En este marco, el proyecto de ley del Distrito presentaría desde mediados de 2012 resistencias por parte de distintos grupos de vecinos organizados de La Boca. Este barrio de residencia de trabajadores, ubicado en el sur de la ciudad junto a la ribera del Riachuelo, se caracteriza por distintos déficits importantes de servicios públicos e infraestructura, además de la proliferación de precarias casas de inquilinato o conventillos distintivos por sus fachadas de colores. En el mismo, coexisten sectores populares y distintas organizaciones sociales con tradición combativa en el marco de una dinámica social más amplia que incluye un circuito turístico, donde se entremezclan sectores populares con artistas independientes y ciertos sectores medios. Distintos vecinos, artistas, trabajadores estatales públicos (maestros, médicos, trabajadores sociales), militantes y miembros de diversas organizaciones comunitarias, sociales, culturales y políticas se fueron movilizando en contra del proyecto oficial que luego terminaría siendo aprobado por la Legislatura de la Ciudad Autónoma de Buenos Aires hacia fines de 2012. Las principales críticas tenían que ver con el tratamiento unilateral de la iniciativa, esto es desde el Estado local y sin participación o discusión vecinal; las posturas que planteaban que lejos de promover el desarrollo artístico o cultural el proyecto tenía como meta favorecer la especulación inmobiliaria y la apropiación de tierras e inmuebles deteriorados para el sector privado (generando incrementos de los alquileres, desalojos y por ende una mayor exclusión social y expulsión de los sectores populares del barrio); el poco estímulo para los vecinos de sectores medios asentados (sin ningún nivel de desgravación fiscal) o los artistas pequeños e independientes, así como centros y organizaciones culturales comunitarias y barriales que no serían priorizadas. ${ }^{11}$

De este modo, los principales ejes por parte de los actores vecinales movilizados frente a este proyecto se dirigían a priorizar el derecho a la vivienda y a un desarrollo urbano más equilibrado, dando cuenta de la importancia del patrimonio y la identidad barrial como cuestiones prioritarias. Si bien la movilización vecinal no pudo impedir la aprobación de de la ley de formación del Distrito de las Artes, la misma incorporaría como temática citada la atención de la situación de vulnerabilidad social en la zona, relevando la necesidad de soluciones habitacionales y acciones dirigidas a facilitar la permanencia de las familias radicadas en el mismo, aunque con pocos resultados tangibles a la vista.

En efecto, el barrio de La Boca desde el año 2006 tiene decretada la emergencia ambiental y urbanística por el propio gobierno porteño. En sucesivas oleadas a través de los

11 Ver (THOMASZ, (2014 y 2016). 
años fue perdiendo distintas manzanas en beneficio de los intereses privados y no logró sumar las viviendas necesarias para los vecinos. Esta tendencia, continuaba hasta la actualidad ${ }^{12}$, era descripta desde una organización social y política: "El Barrio de La Boca viene sufriendo desde hace décadas un grave deterioro de su trama urbana. Esa degradación y sus consecuencias han sido sistemáticamente denunciadas por innumerables Agrupaciones Vecinales, sin encontrar soluciones reales a sus necesidades. Afortunadamente, la construcción de lazos de solidaridad entre los vecinos es muy sólida y hace posible que la ayuda mutua sea más significativa que la respuesta institucional a la hora de pensar en soluciones efectivas. El Barrio de La Boca vive desde hace muchos años en un permanente estado de emergencia habitacional, posee además, un alto nivel de contaminación de los cursos de agua, conjuntamente con una evidente degradación del medio ambiente en general y de la calidad de vida de sus habitantes. Se ha llegado a límites impensables e inaceptables por su gravedad y exigen propuestas para un desarrollo sustentable, y no la cesión de espacio público para negocios privados".

En consecuencia, el Distrito de las Artes lejos de estimular las actividades culturales de tipo comunitario y artístico independiente buscaría favorecer en gran medida la instalación de grandes estructuras de la industria cultural y del entretenimiento (un ejemplo claro es la Usina del Arte, con su oferta de espectáculos más exclusivos e internacionales), con fuerte eje en la promoción del turismo. Ello se sumaría al aprovechamiento ya dado de lugares emblemáticos, como el paseo de Caminito, con viejos conventillos reciclados por inversores y el predominio de una lógica más mercantil de aprovechamiento de la cultura barrial. Al mismo tiempo, la ley del Distrito favorecería claramente a los actores empresariales en lo que refiere a la adquisición de inmuebles, terrenos y la construcción de nueva obras, en detrimento de la población residente con menores recursos. Por ende, no se estaría buscando mayormente impulsar el desarrollo socioeconómico de la población local tanto en términos de mejoras sociales como nuevas fuentes empleo, sino más bien fortalecer un sendero especulativo a nivel inmobiliario y en lo que refiere a la apropiación privada del suelo urbano.

\footnotetext{
${ }^{12}$ La disputa reciente y no concluida respecto a terrenos cercanos a la cancha de Boca Juniors para la construcción de un nuevo estadio del club en la zona de Casa Amarilla (que no prosperó judicialmente por la fuerte oposición de organizaciones vecinales), forma parte de esta dinámica prioritariamente especulativa y de negocio respecto a las tierras del barrio.
} 


\section{CONCLUSIONES}

Los Distritos Económicos aparecen en la CABA como nueva forma de intervención urbana dentro de una dinámica neoliberal más general de producción de la ciudad. Esta perspectiva, con eje en el interés privado y una lógica de acción estatal facilitadora del mercado, resulta predominante en el despliegue de las denominadas industrias creativas y estratégicas como tendencia creciente a nivel urbano global. En efecto, la apertura de nuevos conglomerados culturales e intervenciones artísticas y de carácter estratégico forman parte de un nuevo marketing de ciudades, que se iría extendiendo paulatinamente en distintos lugares del planeta y daría pie a la promoción de la clusterización o concentración territorial de estas industrias.

En este marco, el trabajo buscó examinar los modelos de intervención urbana puestos en discusión con este tipo de iniciativas, así como evidenciar las dificultades para articular los diversos intereses en juego en el contexto porteño de los últimos años. Precisamente, con los Distritos el impulso público se orientó a acondicionar el entorno y apalancar al sector privado, con incentivos fiscales y regulatorios, de modo de integrar áreas menos aprovechadas pero bien ubicadas de la ciudad. Esta idea de producir mercado, tanto del suelo, como a nivel comercial e industrial donde no existía y con fuerte apoyo del Estado, resultó central dentro de una concepción de "ciudad empresarial-privada" por parte de la autoridades de la CABA. En consecuencia, las cuestiones más amplias vinculadas a los impactos sociales de la política y los derechos a ser priorizados quedarían mayormente rezagados.

El Distrito Audiovisual y el Distrito de la Artes, más allá de las particularidades socio territoriales distintivas (ubicación geográfica, desarrollo socioeconómico y cultural), mostrarían tendencias estructurales similares en lo que refiere a la valorización inmobiliaria, el predominio empresarial y la dinámica expulsiva de la población de menores recursos en términos de "gentrificación". Estos procesos adquirirían mayor magnitud en el barrio de La Boca, por su situación de emergencia habitacional, social y ambiental, junto a una trama de organizaciones y diversos actores activos y movilizados frente a las demandas barriales y la utilización de tierras disponibles. Con una correlación de fuerzas desigual en la discusión sobre el Distrito de las Artes, sus intereses gravitarían mucho menos que los de inversores, empresarios y referentes del sector inmobiliario, apoyados a nivel de incentivos y acciones por las autoridades porteñas.

En consecuencia, a partir del análisis desarrollado queda por verse como a futuro en la Ciudad Autónoma de Buenos Aires se desenvolverá esta tensión entre los intereses del sector 
privado con apoyo gubernamental y el de los actores sociales con eje en un enfoque de derechos. La contraposición general (más allá de los matices que puedan darse) entre un modelo de ciudad neoliberal pro-mercado, predominante en distintos lugares, y otro más inclusivo, sobre la base de lo que (LEFEBVRE, 1973) planteaba como derecho a la ciudad y que examinado en la actualidad supone mayor integración, priorización del vínculo social y ejercicio de la ciudadanía, aparecerá como la división básica a partir del cual se sostendrán los principales postulados políticos para pensar el desarrollo urbano en la actualidad.

\section{BIBLIOGRAFÍA}

ARQUEROS, Soledad \& GONZÁLEZ REDONDO, Carolina. La política de distritos del sur de Buenos Aires: una mirada en perspectiva. Quid 16. Revista del Área de Estudios Urbanos, (6), 7-29, 2017.

BORELLO, José \& GONZÁLEZ, Leandro. Características de la producción audiovisual en la Argentina: resultados de una encuesta reciente a productoras. Imagofagia, Nº 6. 2012.

BRENNER, Neil \& THEODORE, Nik (eds.) Spaces of Neoliberalism: Urban Restructuring in North America and West Europe. Chicago: Wiley-Blackwell. 2002.

CARMONA, Rodrigo. El desafío de gobernar ciudades en Argentina. Alcances y perspectivas en materia de organización política y administrativa. Revista de Direito da Cidade, 7(1), 206-225. 2015.

CARRIÓN, Fernando. El desafío político de gobernar la ciudad. Revista Nueva Sociedad, N²12, noviembre-diciembre, 2007. 2005.

El centro histórico como proyecto y objeto de deseo. Revista EURE, v 31, n 93, p. 89-100,

CICCOLELLA, Pablo. Territorios del Capitalismo Global: una agenda para la Geografía actual. En: CICCOLELLA, Pablo (comp.) Metrópolis latinoamericanas: más allá de la globalización, Quito: OLACCHI, 49-70, 2011.

DE MATTOS, Carlos. Globalización y metamorfosis metropolitana en América Latina: de la ciudad a lo urbano generalizado. Revista de Geografía Norte Grande, (47), 81-104. 2010.

Mercado metropolitano de trabajo y desigualdades sociales en el Gran Santiago:¿ Una ciudad dual? EURE (Santiago), 28(85), 51-70, 2002.

DÍAZ, Mariela; FERME, Nicolás y RASPALL, Tomás. Proyectos de renovación urbana en la Ciudad de Buenos Aires. El caso del Distrito Tecnológico de Parque Patricios. En: Sandra Lencioni; Sonia Vidal-Koppmann; Rodrigo Hidalgo y Paulo Xavier Pereira (eds.): Transformaciones socioterritoriales en las metrópolis de Buenos Aires, San Pablo y Santiago, San Pablo. San Pablo: FAUUSP, 2011. 
DI VIRGILIO, Mercedes. La(s) política(s) educativa(s) como objeto de investigación: formas de construcción y de abordaje. En DI VIRGILIO, Mercedes y WAINERMAN, Catalina. El quehacer de la investigación en educación, Buenos Aires, Manantial, 2010.

FLORIDA, Richard. The rise of the creative class: And how it's transforming work, leisure, community and everyday life. Nueva York: Basic Books, 2002.

Cities and the creative class. Nueva York: Routledge, 2005

HARVEY, David. Espacios del capital. Hacia una geografía crítica. Madrid: Akal, 2007.

'From managerialism to entrepreneurialism: the transformation of urban governance in

late capitalism', Geografiska Annaler, Series B: Human geography, 17 B, 1, 3-17, 1989.

GIONCO, Pamela. Distrito audiovisual de la Ciudad Autónoma de Buenos Aires: ¿régimen de promoción o de concentración económica? En: III Congreso Internacional de la Asociación Argentina de Estudios de Cine y Audiovisual (AsAECA), Córdoba, 2012.

GOICOECHEA, María Eugenia. La ciudad de Buenos Aires como ámbito y objeto de negocios. Reflexiones en torno a la gestión urbana del Distrito Tecnológico Parque Patricios. Revista Quid 16, No 4, pp. 161- 185, 2014.

Industrias TIC y nuevos aglomerados productivos en la Ciudad de Buenos Aires. El caso del Distrito tecnológico de Parque Patricios. En: $9^{\circ}$ Bienal del Coloquio de Transformaciones Territoriales, Universidad Nacional de Tucumán, San Miguel de Tucumán, Argentina, 2012.

GONZÁLEZ, Leandro. Política del cine y el audiovisual: entre lo global y lo local, XVIII Jornadas Nacionales de Investigadores en comunicación, 9-11 de Octubre de 2014.

LEFEBVRE, Henri. El derecho a la ciudad. Barcelona: Península, 1973.

MOLLENKOPF, John \& CASTELLS, Manuel (eds). Dual city: restructuring New York. Nueva York, Russel Sage Foundation, 1991.

MONTESINOS, Eduard. La ciudad creativa como utopía y una alternativa a partir de Walter Benjamin. XIV Coloquio Internacional de Geocrítica. Las utopías y la construcción de la sociedad del futuro, 2-7, 2016.

OSZLAK, Oscar \& O’DONNELL, Guillermo. Estado y políticas estatales en América Latina: Hacia una estrategia de investigación, Doc. CEDES/CLACSO, Nㅜ 4, Buenos Aires, 1981.

PÍREZ, Pedro. Actores sociales y gestión de la ciudad. Ciudades, México: RNIU, №28, octubrediciembre, 1995.

Gobernabilidad metropolitana en la Argentina. En: BADÍA, Gustavo \& CARMONA, Rodrigo (comp.) La Gestión Local en la Argentina: situación y perspectivas, UNGS, 2008.

RODRÍGUEZ, María Carla \& DI VIRGILIO, Mercedes. Ciudad de Buenos Aires: políticas urbanas neoliberales, transformaciones socio-territoriales y hábitat popular. Revista de Direito da Cidade, 6(2), 323-347, 2014.

SANTOS, Milton. La naturaleza del espacio: técnica y tiempo, razón y emoción Barcelona, Ariel, 2000. 
SILVEIRA, María Laura. Globalización y territorio usado: imperativos y solidaridades. Cuadernos del CENDES, 25(69), 2008.

SOCOLOFF, Ivana. Presentación Dossier: Una reflexión sobre los distritos creativos y las mutaciones del empresarialismo urbano porteño. Quid 16. Revista del Área de Estudios Urbanos, (6), 1-6, 2017.

SOCOLOFF, Ivana (coord.), COLOMBO BLANCO, Ana.; KITAY, Iván.; MALTZ, Hernán; RULLANSKY, Ignacio.; SEIA, Guadalupe; STIBERMAN, Laura \& URDAMPILLETA, Magdalena. Gobernar Buenos Aires. Un estudio sobre las racionalidades políticas en torno al desarrollo local a partir del caso del Distrito Tecnológico de Parque Patricios (2008-2012). En: VII Jornadas de Sociología, UNLP, La Plata, 2012.

SMITH, Neil. The new urban frontier. Gentrification and revanchist city. Boston: Routledge, 1996.

THEODORE, Nik; PECK, Jamie; BRENNER, Neil. Urbanismo neoliberal: la ciudad y el imperio de los mercados. Revista Temas Sociales, N 66, 2009.

THOMASZ, Ana Gretel. Los nuevos distritos creativos de la Ciudad de Buenos Aires: la conversión del barrio de La Boca en el Distrito de las Artes. EURE, Vol. 42, № 126, pp. 145-167, 2016.

THOMASZ, Ana Gretel. La estetización, la nivelación y el saneamiento como metáforas del cambio urbano en La Boca. Anuario. Antropología Social y Cultural en Uruguay, 12, 93-105, 2014. THOMASZ, Ana Gretel. La estetización, la nivelación y el saneamiento como metáforas del cambio urbano en La Boca. Anuario Antropología Social y Cultural en Uruguay, 12, 93-105, 2014.

ZAPATA, María Cecilia. La política habitacional porteña bajo la lupa, De los programas llave en mano a la autogestión del hábitat, Buenos Aires. URL: https://www.teseopress.com/politicahabitacional, 2017.

Trabalho enviado em 13 de outubro de 2017.

Aceito em 19 de outubro de 2017. 